\title{
Integration of Residue Attributes for Sequence Diversity Characterization of Terpenoid Enzymes
}

\author{
Nelson Kibinge, Shun Ikeda, Naoaki Ono, Md. Altaf-Ul-Amin, and Shigehiko Kanaya \\ Graduate School of Information Science, Nara Institute of Science and Technology, 8916-5 Takayama, Ikoma, Nara 630-0192, Japan \\ Correspondence should be addressed to Shigehiko Kanaya; skanaya@gtc.naist.jp
}

Received 1 November 2013; Accepted 21 February 2014; Published 11 May 2014

Academic Editor: Samuel Kuria Kiboi

Copyright ( 2014 Nelson Kibinge et al. This is an open access article distributed under the Creative Commons Attribution License, which permits unrestricted use, distribution, and reproduction in any medium, provided the original work is properly cited.

\begin{abstract}
Progress in the "omics" fields such as genomics, transcriptomics, proteomics, and metabolomics has engendered a need for innovative analytical techniques to derive meaningful information from the ever increasing molecular data. KNApSAcK motorcycle $\mathrm{DB}$ is a popular database for enzymes related to secondary metabolic pathways in plants. One of the challenges in analyses of protein sequence data in such repositories is the standard notation of sequences as strings of alphabetical characters. This has created lack of a natural underlying metric that eases amenability to computation. In view of this requirement, we applied novel integration of selected biochemical and physical attributes of amino acids derived from the amino acid index and quantified in numerical scale, to examine diversity of peptide sequences of terpenoid synthases accumulated in KNApSAcK motorcycle DB. We initially generated a reduced amino acid index table. This is a set of biochemical and physical properties obtained by random forest feature selection of important indices from the amino acid index. Principal component analysis was then applied for characterization of enzymes involved in synthesis of terpenoids. The variance explained was increased by incorporation of residue attributes for analyses.
\end{abstract}

\section{Background}

Biology and other modern sciences have become data intensive and in fact data-driven biology is now a full-fledged domain of specialization among the life sciences. Due to the amorphous nature of the accruing biological data, various databases have continually been developed to allow systematization [1]. A lot however still needs to be done to characterize these compilations into meaningful information. KNApSAcK database describes species-metabolite relationships, and within the KNApSAcK family we have developed an enzyme-reaction database called KNApSAcK motorcycle DB containing reactions and enzyme peptide sequences based on experimental evidence focusing on secondary metabolic reactions in plants.

Increasingly, the need to analyse data in such repositories has made advanced mathematical and statistical tools a mainstay of bioinformatics in recent years, more so in sequencebased analyses [2]. For molecular sequence data especially proteins, standard alphabetical notation of sequence information may not explicitly capture aspects such as their biochemical and physicochemical properties (BPPs) and may to some extent limit tractability to mathematical analyses. Ideally, computational analyses of the often heterogenous datasets require theoretical representations in forms suitable for various data processing models. This formal representation has been defined as sequence feature coding [3]. There has been no standard method of directly encoding quantifiable BPP of protein sequences hitherto. A key research question has thus been how to quantitatively characterize such data for computation whilst considering these aspects and other sequence metadata [4]. In the present study, we introduce a BPP subset for encoding amino acid residue properties into protein sequences during analyses. We found that this increases the flexibility of computational analyses focusing on facets of biochemical, physical, and evolutionary attributes of sequence data. Integration of BPP is employed in examination of diversity in enzymes related to secondary metabolite pathways, specifically those involved in terpenoid synthesis.

Researchers have proposed schemes to ensure amenability of sequences to computation, but it remains difficult to achieve computational objectivity while maintaining biological interpretability. 5-bit and 3-bit binary feature coding 
of amino acids in peptide sequences acids have been used in studies such as [5]. White and Seffens also used 20-bit transformation for neural network application for translation of proteins [6]. A limitation of binary feature coding is the minimal biological information with respect to amino acid diversity. This is because bit coding does not account for relative similarities or differences between amino acids and neither is it flexible to integration of their BPP; besides, binary notation of highly conserved protein sets may also pose numerical difficulties to probability-based models.

Information theory has also been exploited as an alternative, where mutual information and entropy are estimated by the Shannon-Wiener indexing of amino acid properties $[7,8]$. This way, distance and variation between amino acid units, is estimable and therefore is an improvement over the binary coding method. It, however, does not directly represent characteristic attributes such as polarity, molecular size, and other features of residues.

More recently, the amino acid index (AAindex) database has accumulated published data of amino acid properties [9]. Each index has a set of 20 numerical values of a BPP quantified and published in research literature. Currently, AAindex contains 544 indices describing quantifiable amino acids residue properties. This provides a foundation for BPP feature-coding protein by assigning "scale-measured" attributes of amino acids.

AAindex in its entirety (raw form) is not merited for feature coding since it is highly redundant and has some missing values. Atchley and colleagues proposed index reduction using multivariate factor analysis reducing it to five compressed factors [10]. This methodology is a useful solution for AAindex-based metrication of amino acid residues but factor analysis (FA) reduction complicates biological interpretability in downstream analyses. This is because FA just like principal component analysis (PCA) assumes an underlying linear independence of variables whose coefficients, also called "factors," are a proxy interpretation of AAindex variables [11]. This means that "factors" derived are pseudovariables of the actual original properties and in a way add to complexity of biological interpretation in subsequent downstream steps of sequence analyses.

In light of these challenges in analytical systematization of protein sequences, the present work applies a slightly different variable selection criterion from AAindex for the purpose of encoding BPP information into sequence data. This was achieved by use of random forest (RF) algorithm [12] to reduce redundancy and to maximize amino acid metadata captured in the AAindex. Eight BPP indices describing variability of amino acids were selected based on our experimental results. The derived reduced AAindex (rAAindex) is a subset of the original AAindex after elimination of redundancies. We further integrated the rAAindex in characterization of protein sequence diversity in the KNApSAcK motorcycle DB. The enzymes characterized are involved in secondary metabolic pathways of terpenoids and include monoterpenoid synthases, diterpenoid synthases, triterpenoid synthases, and sesquiterpenoid synthases.

\section{Materials and Methods}

2.1. Amino Acid Index and Random Forest Selection of Biochemical and Physical Properties. Amino acid index (AAindex) is a database of numerical indices describing biochemical and physical attributes of the 20 amino acids [9]. It provides a plausible starting point for interpreting peptide BPPs numerically through its "building blocks": the amino acids. We selected a set of important indices that broadly characterize amino acid BPP variation, where RF algorithm [12] was used for index selection.

We denote by $X$ the set of amino acid indices as the explanatory variables. $N$ denotes the set of amino acids (AA). The categorical predictor variable that best defines the AA population is its qualitative attribute of water interaction; that is, every amino acid is described as either hydrophobic or hydrophilic. We therefore denote $Y$ as describing hydrophobicity or hydrophilicity of an amino acid. The $i$ th amino acid, $n_{i}$, is described by a vector $\left(x_{1}, \ldots, x_{m}, y_{i}\right)$.

Raw AAindex is highly redundant and multicollinear. We initially processed it by removal of indices that had missing values for any amino acid. Redundant indices were eliminated by backward elimination of variables whose correlation coefficient was above a threshold of 0.85. 283 indices were retained for RF variable selection.

Random forest (RF) [12] is a popular algorithm in statistics and bioinformatics for two reasons:

(i) it is a powerful classification and regression tree (CART) tool that generates ensembles of decision trees. RF and other decision tree-based classifiers are nonparametric; that is, they do not assume underlying structure in a dataset and are useful for classification and regression modeling of complex biological data;

(ii) RF implements a mechanism of calculating variable importance scores (VIM) by permutation testing. These measures are useful in feature selection and provide an advantage which we explore in this work.

Besides these two advantages, further application to biological research has been documented in [14]. Detailed mechanism of the RF algorithm is described in $[12,15]$ although a generalized outlook of its concepts is illustrated in Figure 1. RF was implemented for selection of reduced AAindex (rAAindex) consisting of indices describing BPPs explaining the variation of amino acids.

In RF, sampling by bootstrap creates an "out-of-bag" (OOB) sample which is an important feature due to its usefulness in estimation of VIM. These scores are derived by permutation testing of the $\mathrm{OOB}$ data in the error estimation step. VIM scores of index $x_{i}$ are described as the mean error rate over all trees in the RF ensemble. Detailed information on VIM calculation is described elsewhere [12], but for descriptive purposes, we simplify the formal representation of this measure as

$$
\operatorname{VIM}\left(x_{i}\right)=\frac{1}{\text { ntree }} \sum_{1}^{\text {ntree }}\left({\widetilde{\operatorname{err} \cdot \mathrm{OOB}_{\mathrm{t}}}}^{\left.\mathrm{err} \cdot \mathrm{OOB}_{\mathrm{t}}\right)}\right.
$$




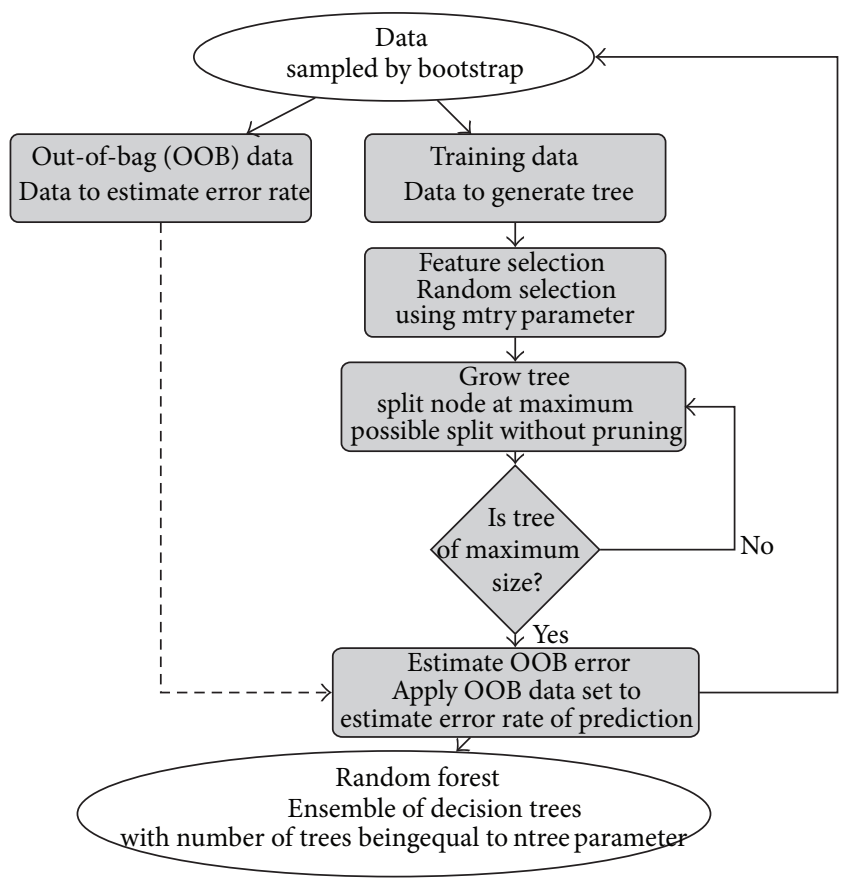

FIGURE 1: Random forest algorithm. Mechanism of the random forest (RF) algorithm starting from the data selection by bootstrapping up to variable importance calculation. The amino acid index data containing physicochemical metrics of amino acids was subjected to RF for index selection.

where $\operatorname{VIM}\left(x_{i}\right)$ is a function estimating the VIM score for variable $x_{i}$ and ntree is the number of trees in the RF ensemble, whereas err. $\widetilde{\mathrm{OOB}}_{\mathrm{t}}$ is the number of misclassifications tested on a tree $t$ where the input was permuted values of variable $x_{i}$. Conversely, err $\cdot \mathrm{OOB}_{\mathrm{t}}$ is the number of misclassifications tested on a tree $t$ whose input was the nonpermuted values of variable $x_{i}$.

Validity of permutation test derivation of VIM in the $\mathrm{RF}$ algorithm operates on the premise that if a variable is "important," then permuting its values (realistically) leads to reduced accuracy of class prediction. Variables were selected using the method described in [16]. The first step involved stochastically running $1000 \mathrm{RF}$ classification trials of AAindex and each time recording the mean decrease in accuracy (VIM score). Indices were then ranked on a decreasing score order. The variation of these VIM scores was obtained and the point of minimum variance was initialized as a threshold, from which 93 amino acid indices were retained for the further index reduction by nested RF feature selection approach described in detail by Genuer et al. [16]. The threshold of significant deviation in the increasing error rates from the nested RF modeling was set to the number of variables above which the error rates significantly increase above the threshold of 0.02 percent meaning that at most a single amino acid misclassification could be accepted from the nested RF. A reduced amino acid index (rAAindex) was thus derived and its usefulness as a representation of amino acid information was tested on data from our KNApSAcK motorcycle database.

\subsection{KNApSAcK Motorcycle DB: Peptide Sequence-Metabolic} Reaction Relationship DB. It is necessary to extend the species-metabolite relationship DB by incorporating a secondary metabolite pathway DB that includes pathways with detected enzymatic reactions and other actual or predicted peptide sequences that may be involved in these pathways. We surveyed reactions of secondary metabolites in scientific literature, and amino acid sequences involved in secondary metabolism were obtained from public databases in PubMed.gov (http://www.ncbi.nlm.nih.gov/). All the data comprising 2,881 secondary metabolic reactions was accumulated in the KNApSAcK motorcycle DB (http://kanaya.naist.jp/motorcycle/top2.html) as shown in the main window of the KNApSAcK motorcycle DB (Figure 2); enzyme reactions can be retrieved using keywords of enzymes, species, genes, metabolites, and peptide sequences obtained from a BLASTP search. For metabolite search using its keywords, we obtain information on enzyme name, reaction involved, compound class (C-class in Figure 2) and subclass (C-subclass) of metabolic reactions, and reaction mechanisms. For BLASTP search, we can predict reaction equations for a targeted peptide sequence using information on the class and subclass of metabolic pathways (Figure 1(c)). Thus, the motorcycle DB makes it possible to predict enzyme reactions based on the class and subclass of metabolic reactions evidenced by experiments mentioned in scientific literature. This differentiates it from KEGG [17] and BioCyc [18]. We have thus far obtained 596,974 protein sequences of 59,165 plant species and 124,292 protein sequences of 66 bacterial species from the nonredundant protein sequences of PlantGDB.

For analytical purposes of the presently developed method, we narrowed our test dataset to terpenoid synthase peptide sequences with $>200$ amino acid residues. Terpenoids are organic metabolites of plants that have been shown to have insect-pesticide properties among other roles. Terpenoid synthases sequence from the KNApSAcK database [19] was examined for patterns in diversity. Enzymes annotated to four families, namely, monoterpenoid synthases, diterpenoid synthases, triterpenoid synthases, and sesquiterpenoid synthases, were examined by PCA. Understanding the data structure of these terpenoid enzyme subfamilies is important for annotation of similar organic compounds [20]. Multiple sequence alignment and gap removal were carried out to extract homologous regions of sequences from the four subfamilies of terpenoid synthases. These domains had a length of 28 residues for the 283 sequences. Binary and rAAindex (BPP) feature coding of amino acid residues in these sequences were compared.

\subsection{Sequence Diversity Characterization Based on Principal} Component Analyses (PCA). The present work attempts to examine diversity of secondary metabolic enzyme groups using datasets from the KNApSAcK motorcycle DB by 


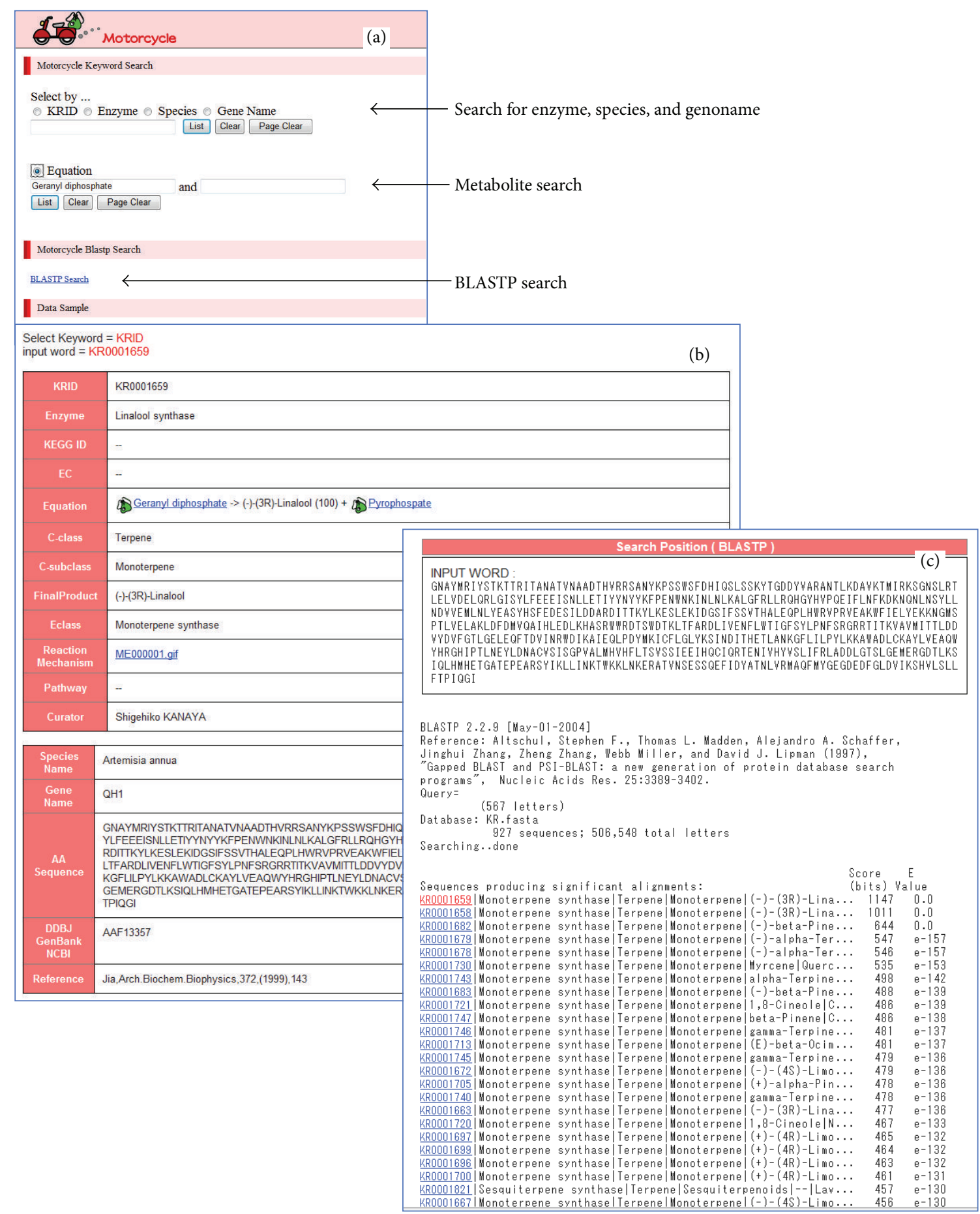

FIGURE 2: KNApSAcK motorcycle database. Enzyme-reaction database. (a) The main window of motorcycle. (b) An example of a keyword search. (c) An example of BLASTP search.

integrating amino acid attributes represented in the rAAindex where PCA was used to analyse variation. PCA is a technique that enables efficient interpretation of variation and relationship between variables in a huge dataset represented by higher dimensional vectors [21]. It is widely applied in bioinformatics as exemplified by Tatusov et al. who phylogenetically classified genomes by protein function
[22]. For comparative purposes, a BPP integrated dataset was analysed in comparison to 8-bit binary-coded enzyme set. We initially generated lattices representing individual sequences and encoded by both rAAindex and the commonly used 8bit binary feature coding. We hypothesized that the BPPencoded set explains more variance of sequences and thus reflects the diversity of proteins based on BPP integration. 


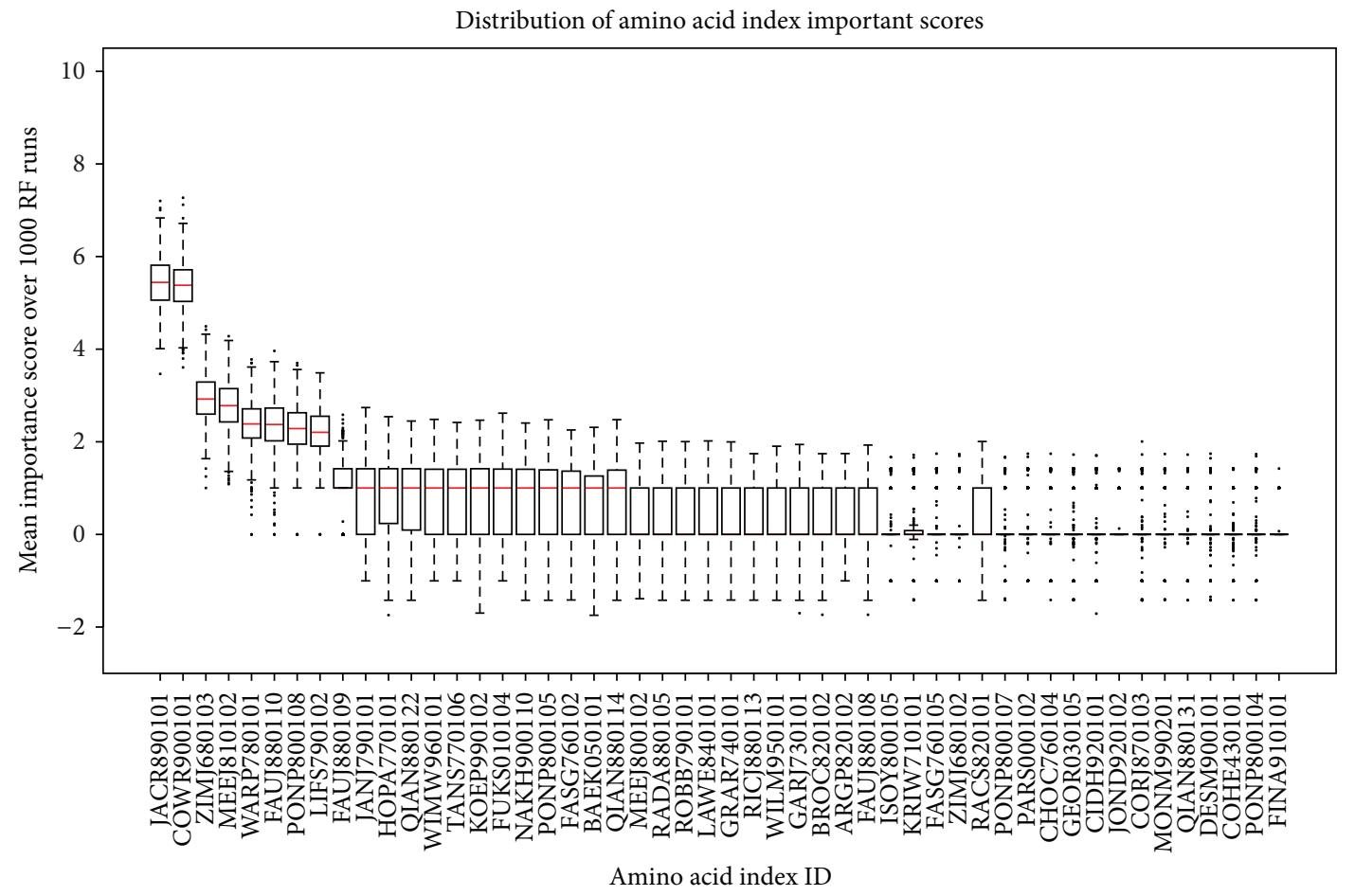

FIGURE 3: Variable importance scores (1000 RF trials). Variable importance score distribution for 1000 runs of random forest classification on the amino acid index. Each boxplot represents distribution of each property (also called variable) represented on the horizontal axis. The properties have been ordered in decreasing order of the median score (red line in boxplot). For easier visualization, the set has been truncated to show the top 50 properties. The corresponding properties are shown in Table 1.

\section{Results and Discussion}

3.1. Reduced Amino Acid Index. Physicochemical properties of amino acids quantitatively describe the overall biochemical behaviour of peptide and protein sequences [13]. The amino acid index database [9] has collected properties of amino acids measured by various researchers since the 1970s using scientific instruments and quantifiable metrics. It is essential to consider these properties in objective analyses of sequence data. Numeric quantification is also pivotal because it gives a flexible way of integrating this information in a mathematically and statistically amenable form different from the alphabetical string representation.

The AAindex database is highly redundant and has some missing values for certain properties. In its raw form, the AAindex is unsuitable for direct BPP encoding. Ideally, a reduced set would work for most sequence analyses. Researchers have utilized various compression techniques to reduce the AAindex. Atchley and colleagues used a multivariate factor analysis to propose a compressed variable set of five vectors describing amino acids in a multidimensional space [10]. More recently, fuzzy c-means algorithm has been applied in clustering the AAindex indices and the resultant clusters incorporated in a support vector machine modeling experiment to predict DNA-binding domains [23].

Factor analysis (FA) is a useful approach for the same purpose of defining a minimal set of "factors" that simplify interpretation of protein sequence characteristics. Random forest (RF) variable reduction differs from FA since RF selects important variables without compressing the whole variable set into fewer descriptive factors. RF has been proven to be a useful tool for biological data as described in [2426]. Here, BPP selection entails minimizing the original variables in the AAindex by elimination of redundancy, high collinearity, and less informative variables whilst maintaining a sufficiently parsimonious set of the original BPP properties represented in the AAindex. Compression (as in FA) on the other hand is redefinition of original AAindex variables into new components by multivariate techniques such as PCA [21] and factor analyses [27]. We argue against compression in the context of AAindex data that while a minimized descriptive set is achieved, there results a complexity of biological interpretation that arises if the redefined variables are applied in subsequent downstream mathematical or statistical analyses.

From the initial 544 properties contained in the amino acid index database, 13 which had missing values were dropped. Initially, the redundancy was further reduced by dropping variables with a correlation coefficient greater than 0.85 , further trimming the set to 283 amino acid indices. The retained indices were then subjected to the RF algorithm (1000 trials) and variable reduction was done using the technique in [16]. Variable importance scores (VIM) were ranked in decreasing order (Figure 3). The "importance" score of a BPP illustrates its significance with regard to amino acid classification. It shows the VIM score distribution for 1000 runs of random forest classification of BPP in the amino acid 


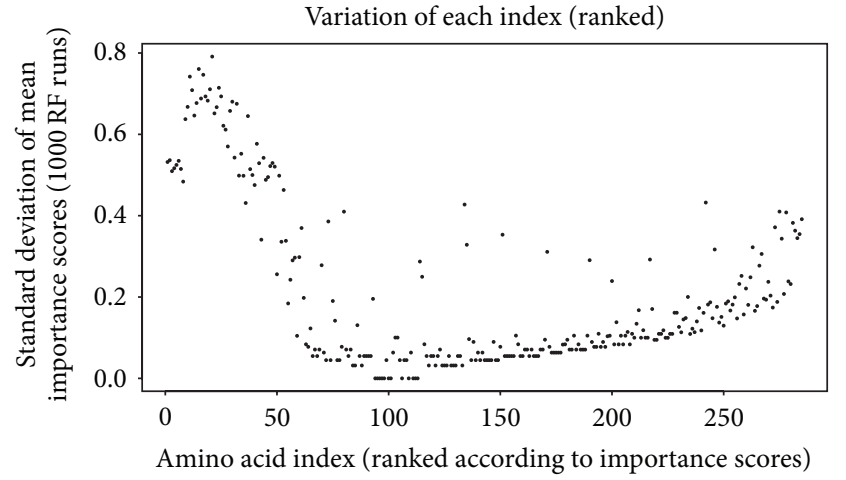

FIGURE 4: Variation of the BPP importance. Standard deviation of the importance scores of the properties ( $y$-axis); models contribution of each property to the performance of the RF algorithm. Those variables with a close to zero variation are less "important." At the tail end, variance is more than zero due to chance.

index. Each boxplot in the figure represents distribution of each BPP (also called variable) represented on the horizontal axis. These properties have been ordered in decreasing order of the median score (red line in boxplot). For easier visualization, the set in the figure has been truncated to show the top 50 properties by mean VIM ranking. The corresponding properties are shown in Table 1. Variation (standard deviation) of the ranked scores was observed as shown in Figure 4. Standard deviation of the importance scores of the properties ( $y$-axis) models contribution of each property towards performance of the RF algorithm. Those variables with a close to zero variation are less "important." At the tail end variance is higher than zero but is large due to chance ( $P$ value $>0.05)$. Variables with a mean VIM score of 0 or less were dropped, lowering the retained variables to 93 indices. Nested RF modeling was done using the ranked indices, by starting with the highest ranked variable and subsequent addition remaining after each step. Error rates were estimated for each step of the nested modeling. Details of nested RF are explained in [16]. The threshold of acceptable error rate was set to 2 percent. Figure 5 shows that when the first 8 indices are used for classification, the error rate remains under the 2 percent error rate (horizontal red line in the figure) threshold, whereas it significantly rises with the addition of subsequent indices.

An RF-reduced subset of the amino acid index, rAAindex, (Table 2) with these 8 most important BPPs, is proposed for use in BPP encoding especially for statistical learning and other mathematical tasks involving protein sequences. The properties retained are shown in Table 3.

\subsection{Characterization of Terpene Synthase Sequence Diversity.} Terpenes are the largest group of plant natural products with a variety of core chemical structures comprising at least 30,000 compounds and synthesized by terpenoid synthases [28]. Terpene diversity is caused by the large number of different terpene synthases used in the first step of terpene synthesis, and some terpene synthases produce multiple products

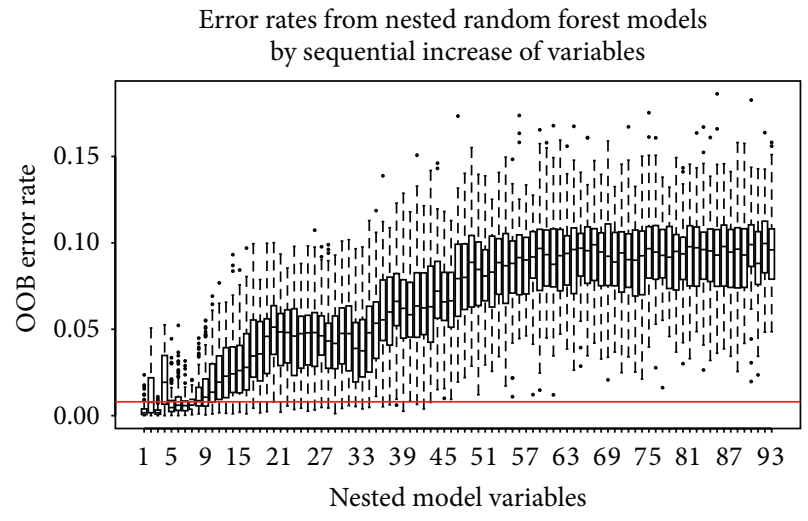

FIGURE 5: Nested random forest error rates. Nested random forest variable selection: variables have been ordered by their importance scores, new RF models are built by single variable addition in the nested RF setup, and RF error rates are measured. In this experiment, each box and whisker plot represents the distribution of error rates from 100 trials at each nested RF step. In total, there were 93 steps corresponding to the 93 top indices from previous step. The $y$-axis shows the error rates as a percentage. The threshold of acceptable mean error rate was set at 2 percent shown by the red horizontal line.

[29]. Terpene synthases are generally classified according to the number of carbons in their substrates, that is, geranyl diphosphate (C10, GPP) for monoterpene synthases, farnesyl diphosphate (C15, FPP) for sesquiterpene synthases, geranylgeranyl diphosphate (C20, GGPP) for diterpene synthases, and squalene for triterpene synthases (C30). The rather limited similarity of plant terpenoids [20] complicates annotation of their enzymes. Clustering algorithms improve the resolution to some extent. PCA was used to analyse variation among 4 terpene synthase subfamilies. We examined the performance of PCA classification when rAAindex BPP encoding is implemented, relative to the commonly used 8bit binary encoding. The combined variance explained by the first two principal components is 30.02 (Figure 6 left) percent, whereas the variance explained by the first two components in binary encoded set is 14.84 percent (Figure 6 right). Triterpenoid synthases can be clearly distinguished from the other categories, which was also consistent with our previous findings [30].

We compared the performance of rAAindex to 8-bit binary encoding of amino acids [31] in an actual dataset (described in the data and methods section). Binary coding is the most popular representation scheme for machine learning tasks of protein data $[5,6]$ and was utilized as a benchmark of comparison to rAAindex encoding. Figure 6 (left) shows the fragment distribution of terpene synthases coded by 8-bit binary notation mainly clustered into five regions. Similarly, Figure 6 (right) shows the distribution of terpene synthases coded by rAAindex into the same 5 groups. Principal component analysis was performed on terpenoid synthase sequence subfamilies, where amino acid residues were encoded in 8-bit binary code. PC1 and PC2 show a combined variance of 14.84 percent explained variance. The second part 
TABLE 1: Top 50 ranked indices. Descriptions of the top 50 indices by ranking of the VIM scores prior to nested RF. The first column represents the AAindex access ID, while the second is the corresponding BPP. Please refer to the amino acid index database in the Supplementary Material available online at http://dx.doi.org/10.1155/2014/753428 for the references in column 2.

\begin{tabular}{|c|c|c|}
\hline & ID & Property \\
\hline 1 & RADA880101 & Information value for accessibility; average fraction 35\% (Biou et al., 1988) \\
\hline 2 & ROSM880101 & Information value for accessibility; average fraction 23\% (Biou et al., 1988) \\
\hline 3 & KIDA850101 & Retention coefficient in TFA (Browne et al., 1982) \\
\hline 4 & EISD840101 & Normalized hydrophobicity scales for alpha + beta-proteins (Cid et al., 1992) \\
\hline 5 & JACR890101 & Normalized hydrophobicity scales for alpha/beta-proteins (Cid et al., 1992) \\
\hline 6 & COWR900101 & Consensus normalized hydrophobicity scale (Eisenberg, 1984) \\
\hline 7 & BLAS910101 & Direction of hydrophobic moment (Eisenberg-McLachlan, 1986) \\
\hline 8 & MEEJ810101 & Hydrophobic parameter pi (Fauchere-Pliska, 1983) \\
\hline 9 & CIDH920104 & Number of hydrogen bond donors (Fauchere et al., 1988) \\
\hline 10 & GRAR740102 & Number of full nonbonding orbitals (Fauchere et al., 1988) \\
\hline 11 & ZIMJ680103 & Polarity (Grantham, 1974) [13] \\
\hline 12 & MEEJ810102 & Hydration number (Hopfinger, 1971), Cited by Charton-Charton (1982) \\
\hline 13 & RADA880104 & Hydropathy index (Kyte-Doolittle, 1982) \\
\hline 14 & KUHL950101 & Hydrophobic parameter (Levitt, 1976) \\
\hline 15 & FAUJ880110 & Conformational preference for parallel beta-strands (Lifson-Sander, 1979) \\
\hline 16 & RADA880107 & Average surrounding hydrophobicity (Manavalan-Ponnuswamy, 1978) \\
\hline 17 & WARP780101 & Retention coefficient in $\mathrm{NaClO} 4$ (Meek-Rossetti, 1981) \\
\hline 18 & BIOV880102 & Retention coefficient in NaH2PO4 (Meek-Rossetti, 1981) \\
\hline 19 & BIOV880101 & 8 A contact number (Nishikawa-Ooi, 1980) \\
\hline 20 & FASG890101 & Partition coefficient (Pliska et al., 1981) \\
\hline 21 & PONP800108 & Average number of surrounding residues (Ponnuswamy et al., 1980) \\
\hline 22 & FAUJ830101 & Hydrophobicity (Prabhakaran, 1990) \\
\hline 23 & CORJ870101 & Weights for beta-sheet at the window position of 2 (Qian-Sejnowski, 1988) \\
\hline 24 & MANP780101 & Transfer-free energy from chx to wat (Radzicka-Wolfenden, 1988) \\
\hline 25 & LIFS790102 & Transfer-free energy from chx to oct (Radzicka-Wolfenden, 1988) \\
\hline 26 & GUOD860101 & Energy transfer from out to in (95\% buried) (Radzicka-Wolfenden, 1988) \\
\hline 27 & WOLS870101 & Mean polarity (Radzicka-Wolfenden, 1988) \\
\hline 28 & WOLR790101 & Side chain hydropathy, uncorrected for solvation (Roseman, 1988) \\
\hline 29 & LEVM760101 & Side chain hydropathy, corrected for solvation (Roseman, 1988) \\
\hline 30 & WOLR810101 & Normalized frequency of chain reversal D (Tanaka-Scheraga, 1977) \\
\hline 31 & ROSM880102 & Average interactions per side chain atom (Warme-Morgan, 1978) \\
\hline 32 & WOEC730101 & Polar requirement (Woese, 1973) \\
\hline 33 & PLIV810101 & Hydration potential (Wolfenden et al., 1981) \\
\hline 34 & RADA880108 & Principal property value zl (Wold et al., 1987) \\
\hline 35 & FAUJ880109 & Polarity (Zimmerman et al., 1968) \\
\hline 36 & HOPA770101 & Free energies of transfer of AcWl-X-LL peptides from bilayer interface to water (Wimley-White, 1996) \\
\hline 37 & CIDH920103 & $\begin{array}{l}\text { Hydropathy scale based on self-information values in the two-state model ( } 9 \% \text { accessibility) (Naderi-Manesh } \\
\text { et al., 2001) }\end{array}$ \\
\hline 38 & TANS770106 & $\begin{array}{l}\text { Hydropathy scale based on self-information values in the two-state model (16\% accessibility) } \\
\text { (Naderi-Manesh et al., 2001) }\end{array}$ \\
\hline 39 & PRAM900101 & Hydrophilicity scale (Kuhn et al., 1995) \\
\hline 40 & ENGD860101 & Retention coefficient at pH 2 (Guo et al., 1986) \\
\hline 41 & BROC820101 & Modified Kyte-Doolittle hydrophobicity scale (Juretic et al., 1998) \\
\hline 42 & NADH010103 & Knowledge-based membrane-propensity scale from 1D_Helix in MPtopo databases (Punta-Maritan, 2003) \\
\hline 43 & NADH010102 & Hydrophobicity index (Wolfenden et al., 1979) \\
\hline
\end{tabular}


TABLE 1: Continued.

\begin{tabular}{lll}
\hline \multicolumn{1}{c}{ ID } & Property \\
\hline 44 & KYTJ820101 & Hydrophobicity-related index (Kidera et al., 1985) \\
45 & EISD860103 & Weights from the IFH scale (Jacobs-White, 1989) \\
46 & NISK800101 & Hydrophobicity index, 3.0 pH (Cowan-Whittaker, 1990) \\
47 & JURD980101 & Scaled side chain hydrophobicity values (Black-Mould, 1991) \\
48 & WIMW960101 & NNEIG index (Cornette et al., 1987) \\
49 & QIAN880122 & Hydrophobicity index (Engelman et al., 1986) \\
50 & PUNT030101 & Hydrophobicity index (Fasman, 1989) \\
\hline
\end{tabular}

TABLE 2: Reduced Amino Acid Index (rAAindex). The first column contains the names of the 20 amino acids that make up protein sequences. Each of the 8 subsequent columns is a BPP attribute selected based on its importance in explaining variation of the amino acid. Each row is a vector describing an amino acid in 8 dimensions, each of which represents a physical property tabulated in Table 3.

\begin{tabular}{|c|c|c|c|c|c|c|c|c|}
\hline Amino acid & JACR890101 & COWR900101 & ZIMJ680103 & MEEJ810102 & FAUJ880110 & WARP780101 & PONP800108 & LIFS790102 \\
\hline Ala & 0.18 & 0.42 & 0.00 & 1.00 & 0.00 & 10.04 & 6.05 & 1.00 \\
\hline Arg & -5.40 & -1.56 & 52.00 & -2.00 & 3.00 & 6.18 & 5.70 & 0.68 \\
\hline Asn & -1.30 & -1.03 & 3.38 & -3.00 & 3.00 & 5.63 & 5.04 & 0.54 \\
\hline Asp & -2.36 & -0.51 & 49.70 & -0.50 & 4.00 & 5.76 & 4.95 & 0.50 \\
\hline Cys & 0.27 & 0.84 & 1.48 & 4.60 & 0.00 & 8.89 & 7.86 & 0.91 \\
\hline Gln & -1.22 & -0.96 & 3.53 & -2.00 & 3.00 & 5.41 & 5.45 & 0.28 \\
\hline Glu & -2.10 & -0.37 & 49.90 & 1.10 & 4.00 & 5.37 & 5.10 & 0.59 \\
\hline Gly & 0.09 & 0.00 & 0.00 & 0.20 & 0.00 & 7.99 & 6.16 & 0.79 \\
\hline His & -1.48 & -2.28 & 51.60 & -2.20 & 1.00 & 7.49 & 5.80 & 0.38 \\
\hline Ile & 0.37 & 1.81 & 0.13 & 7.00 & 0.00 & 8.72 & 7.51 & 2.60 \\
\hline Leu & 0.41 & 1.80 & 0.13 & 9.60 & 0.00 & 8.79 & 7.37 & 1.42 \\
\hline Lys & -2.53 & -2.03 & 49.50 & -3.00 & 1.00 & 4.40 & 4.88 & 0.59 \\
\hline Met & 0.44 & 1.18 & 1.43 & 4.00 & 0.00 & 9.15 & 6.39 & 1.49 \\
\hline Phe & 0.50 & 1.74 & 0.35 & 12.60 & 0.00 & 7.98 & 6.62 & 1.30 \\
\hline Pro & -0.20 & 0.86 & 1.58 & 3.10 & 0.00 & 7.79 & 5.65 & 0.35 \\
\hline Ser & -0.40 & -0.64 & 1.67 & -2.90 & 2.00 & 7.08 & 5.53 & 0.70 \\
\hline Thr & -0.34 & -0.26 & 1.66 & -0.60 & 2.00 & 7.00 & 5.81 & 0.59 \\
\hline $\operatorname{Trp}$ & -0.01 & 1.46 & 2.10 & 15.10 & 0.00 & 8.07 & 6.98 & 0.89 \\
\hline Tyr & -0.08 & 0.51 & 1.61 & 6.70 & 2.00 & 6.90 & 6.73 & 1.08 \\
\hline Val & 0.32 & 1.34 & 0.13 & 4.60 & 0.00 & 8.88 & 7.62 & 2.63 \\
\hline
\end{tabular}

TABLE 3: Annotation of the selected properties. Descriptions of the 8 indices selected after the nested random forest variable selection. The first column represents the AAindex access ID, while the second is the corresponding BPP. Please refer to the amino acid index database for the references in column 2.

\begin{tabular}{lcc}
\hline & ID & Property \\
\hline 1 & JACR890101 & Number of full nonbonding orbitals (Fauchere et al., 1988) \\
2 & COWR900101 & Conformational preference for parallel beta-strands (Lifson-Sander, 1979) \\
3 & ZIMJ680103 & Retention coefficient in NaH2PO4 (Meek-Rossetti, 1981) \\
4 & MEEJ810102 & Average number of surrounding residues (Ponnuswamy et al., 1980) \\
5 & WARP780101 & Average interactions per side chain atom (Warme-Morgan, 1978) \\
6 & FAUJ880110 & Polarity (Zimmerman et al., 1968) \\
7 & PONP800108 & Weights from the IFH scale (Jacobs-White, 1989) \\
8 & LIFS790102 & Hydrophobicity index, 3.0 pH (Cowan-Whittaker, 1990) \\
\hline
\end{tabular}

of the figure illustrates PCA of the same data set encoding the biochemical and physical properties of amino acid residues described above. PC1 and PC2 in this case explain 30.02 percent variance showing that more sequence information is described by the BPP subset. The four subfamilies clustered are monoterpenoid, diterpenoid, triterpenoid, and sesquiterpenoid synthases. Triterpenoid synthases and subgroups of terpenoid synthases are distinctly different in structure compared to the other synthases. It is noted that three types of terpene synthases except for the diterpene synthases are 


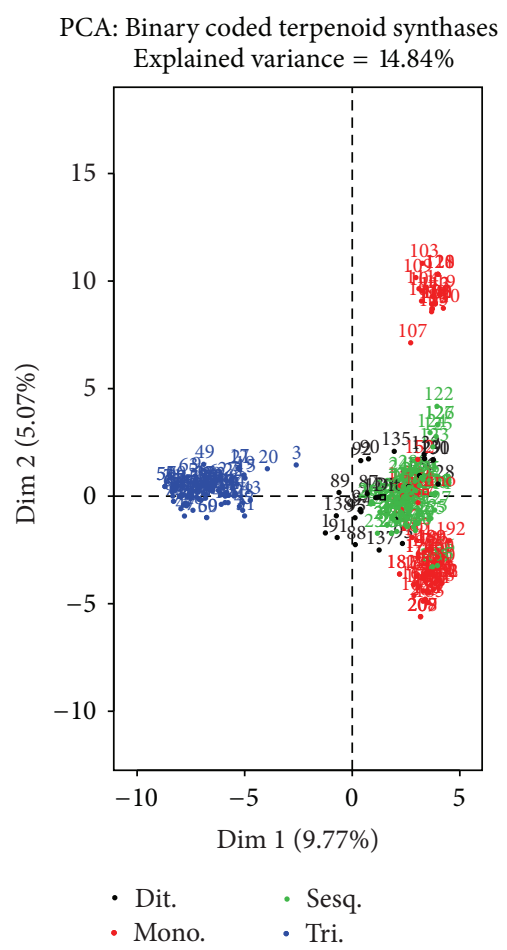

(a)

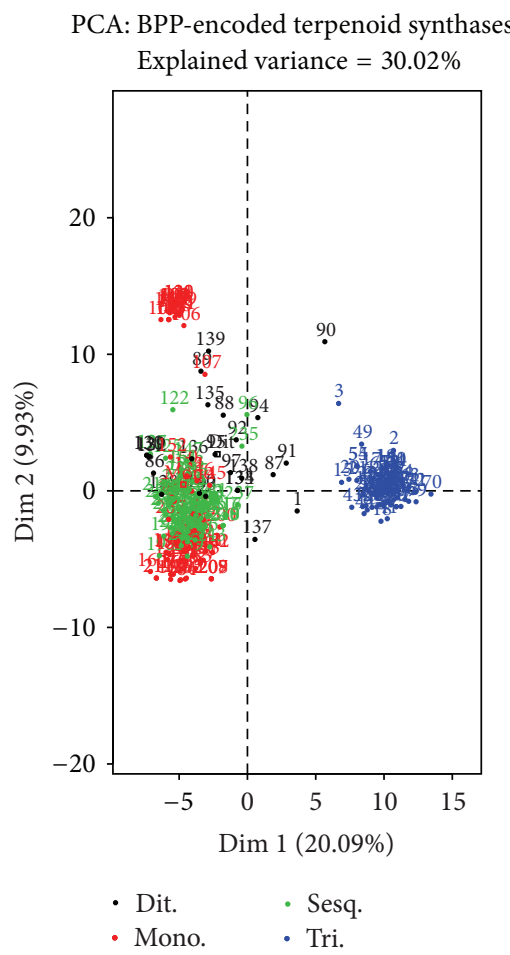

(b)

FIGURE 6: Principal component analysis of terpenoid synthases. Left: principal component analysis of the terpenoid synthase subfamilies where amino acid residues are encoded in 8-bit binary method. PC1 and PC2 show a combined variance of 14.84 percent explained variance. Right: principal component analysis of the same data set encoded using the biochemical and physical properties of amino acid residues. PC1 and PC2 in this case explain 30.02 percent variance. The four subfamilies clustered are monoterpenoid, diterpenoid, triterpenoid, and sesquiterpenoid synthases. Triterpenoid synthases and subgroups of terpenoid synthases are distinctly different in structure compared to the other synthases.

less divergent at the peptide sequence level; that is, small changes in peptide sequences of the terpene synthase make it possible to synthesize many different terpenoid compounds. The orders of fragments from the $\mathrm{N}$ - to the $\mathrm{C}$-terminus in enzymes are arranged in two clusters for monoterpene synthases and are a single cluster for the other categories. Thus, monoterpene and sesquiterpene synthases are very similar in arrangement of peptide fragments, which is consistent with the similarity of the 3D structures in monoterpene and sesquiterpene synthases $[32,33]$ and with the fact that several bifunctional enzymes possess both sesquiterpene and monoterpene synthase activities [33,34].

Diterpene and triterpene synthases have inherent structures specified by a single cluster for diterpene synthases and two clusters from the $\mathrm{N}$ - to C-terminus for triterpene synthases.

\section{Conclusion}

This paper has introduced a subset of eight biochemical and physical attributes of amino acids that can be encoded in protein sequences for sequence-based analysis. These features quantify attributes of individual residues in numerical metrics that improve amenability to mathematical and statistical tasks and also enhance biological interpretability of such tasks. Terpenoid synthases protein set was used to evaluate the encoding of these attributes by PCA. The terpenoid synthase subfamilies established that more variance is explained when BPPs are encoded compared to the commonly used binary encoding which does not integrate physicochemical aspects of protein sequences.

\section{Conflict of Interests}

The authors declare that there is no financial interest or conflict of interests regarding the publication of this paper.

\section{Acknowledgments}

This work was supported by the National Bioscience Database Center, Japan, and the Ministry of Education, Culture, Sports, Science, and Technology of Japan (Grant-in-Aid for Scientific Research on Innovation Areas "Biosynthetic Machinery: Deciphering and Regulating the System for Creating Structural Diversity of Bioactivity Metabolites (2007)"). 


\section{References}

[1] L. D. Stein, "Integrating biological databases," Nature Reviews Genetics, vol. 4, no. 5, pp. 337-345, 2003.

[2] M. He and S. Petoukhov, Mathematics of Bioinformatics: Theory, Methods and Applications, vol. 19, John Wiley \& Sons, 2011.

[3] L. Kong, Y. Zhang, Z.-Q. Ye et al., "CPC: assess the proteincoding potential of transcripts using sequence features and support vector machine," Nucleic acids research, vol. 35, supplement 2, pp. W345-W349, 2007.

[4] M. Vendruscolo and G. G. Tartaglia, "Towards quantitative predictions in cell biology using chemical properties of proteins," Molecular BioSystems, vol. 4, no. 12, pp. 1170-1175, 2008.

[5] A. Coghlan, D. A. Mac Dónaill, and N. H. Buttimore, "Representation of amino acids as five-bit or three-bit patterns for filtering protein databases," Bioinformatics, vol. 17, no. 8, pp. 676-685, 2001.

[6] G. White and W. Seffens, "Using a neural network to backtranslate amino acid sequences," Electronic Journal of Biotechnology, vol. 1, no. 3, pp. 17-18, 1998.

[7] S. Henikoff and J. G. Henikoff, "Amino acid substitution matrices from protein blocks," Proceedings of the National Academy of Sciences of the United States of America, vol. 89, no. 22, pp. 10915-10919, 1992.

[8] O. Weiss, M. A. Jiménez-Montaño, and H. Herzel, "Information content of protein sequences," Journal of Theoretical Biology, vol. 206, no. 3, pp. 379-386, 2000.

[9] S. Kawashima and M. Kanehisa, "AAindex: amino acid index database," Nucleic Acids Research, vol. 28, no. 1, p. 374, 2000.

[10] W. R. Atchley, J. Zhao, A. D. Fernandes, and T. Drüke, "Solving the protein sequence metric problem," Proceedings of the National Academy of Sciences of the United States of America, vol. 102, no. 18, pp. 6395-6400, 2005.

[11] W. J. Krzanowski, Principles of Multivariate Analysis, Oxford University Press, 2000.

[12] L. Breiman, "Random forests," Machine Learning, vol. 45, no. 1, pp. 5-32, 2001.

[13] R. Grantham, "Amino acid difference formula to help explain protein evolution," Science, vol. 185, no. 4154, pp. 862-864, 1974.

[14] A. L. Boulesteix, S. Janitza, J. Kruppa, and I. R. Konig, "Overview of random forest methodology and practical guidance with emphasis on computational biology and bioinformatics," Wiley Interdisciplinary Reviews: Data Mining and Knowledge Discovery, vol. 2, no. 6, pp. 493-507, 2012.

[15] V. Svetnik, A. Liaw, C. Tong, J. Christopher Culberson, R. P. Sheridan, and B. P. Feuston, "Random forest: a classification and regression tool for compound classification and QSAR modeling," Journal of Chemical Information and Computer Sciences, vol. 43, no. 6, pp. 1947-1958, 2003.

[16] R. Genuer, J.-M. Poggi, and C. Tuleau-Malot, "Variable selection using random forests," Pattern Recognition Letters, vol. 31, no. 14, pp. 2225-2236, 2010.

[17] M. Kanehisa and S. Goto, "KEGG: kyoto encyclopedia of genes and genomes," Nucleic Acids Research, vol. 28, no. 1, pp. 27-30, 2000.

[18] R. Caspi, H. Foerster, C. A. Fulcher et al., "The MetaCyc Database of metabolic pathways and enzymes and the BioCyc collection of pathway/genome databases," Nucleic Acids Research, vol. 36, no. 1, pp. D623-D631, 2008.

[19] Y. Shinbo, Y. Nakamura, M. Altaf-Ul-Amin et al., "KNApSAcK: a comprehensive species-metabolite relationship database," in Plant Metabolomics, pp. 165-181, Springer, 2006.
[20] J. Bohlmann, G. Meyer-Gauen, and R. Croteau, "Plant terpenoid synthases: molecular biology and phylogenetic analysis," Proceedings of the National Academy of Sciences of the United States of America, vol. 95, no. 8, pp. 4126-4133, 1998.

[21] I. Jollie, Principal Component Analysis, Wiley Online Library, 2005.

[22] R. L. Tatusov, D. A. Natale, I. V. Garkavtsev et al., “The COG database: new developments in phylogenetic classification of proteins from complete genomes," Nucleic Acids Research, vol. 29, no. 1, pp. 22-28, 2001.

[23] H.-L. Huang, I.-C. Lin, Y.-F. Liou et al., "Predicting and analyzing DNA-binding domains using a systematic approach to identifying a set of informative physicochemical and biochemical properties," BMC Bioinformatics, vol. 12, no. 1, article S47, 2011.

[24] R. Díaz-Uriarte and S. Alvarez de Andrés, "Gene selection and classification of microarray data using random forest," $B M C$ Bioinformatics, vol. 7, article 3, 2006.

[25] M. Sandri and P. Zuccolotto, "Variable selection using random forests," in Data Analysis, Classification and the Forward Search, pp. 263-270, Springer, 2006.

[26] C. Strobl, A.-L. Boulesteix, T. Kneib, T. Augustin, and A. Zeileis, "Conditional variable importance for random forests," $B M C$ Bioinformatics, vol. 9, article 307, 2008.

[27] P. Kline, An Easy Guide to Factor Analysis, Routledge, 1994.

[28] J. D. Connolly and R. A. Hill, Dictionary of Terpenoids. 1. Monoand Sesquiterpenoids, vol. 1, CRC Press, 1991.

[29] J. Degenhardt, T. G. Köllner, and J. Gershenzon, "Monoterpene and sesquiterpene synthases and the origin of terpene skeletal diversity in plants," Phytochemistry, vol. 70, no. 15-16, pp. 16211637, 2009.

[30] S. Ikeda, T. Abe, Y. Nakamura et al., "Systematization of the protein sequence diversity in enzymes related to secondary metabolic pathways in plants, in the context of big data biology inspired by the KNApSAcK Motorcycle database," Plant and Cell Physiology, vol. 54, no. 5, pp. 711-727, 2013.

[31] R. Staden, "Sequence data handling by computer," Nucleic Acids Research, vol. 4, no. 11, pp. 4037-4051, 1977.

[32] D. C. Hyatt, B. Youn, Y. Zhao et al., "Structure of limonene synthase, a simple model for terpenoid cyclase catalysis," Proceedings of the National Academy of Sciences of the United States of America, vol. 104, no. 13, pp. 5360-5365, 2007.

[33] D. A. Nagegowda, M. Gutensohn, C. G. Wilkerson, and N. Dudareva, "Two nearly identical terpene synthases catalyze the formation of nerolidol and linalool in snapdragon flowers," Plant Journal, vol. 55, no. 2, pp. 224-239, 2008.

[34] N. J. Nieuwenhuizen, M. Y. Wang, A. J. Matich et al., “Two terpene synthases are responsible for the major sesquiterpenes emitted from the flowers of kiwifruit (Actinidia deliciosa)," Journal of Experimental Botany, vol. 60, no. 11, pp. 3203-3219, 2009. 

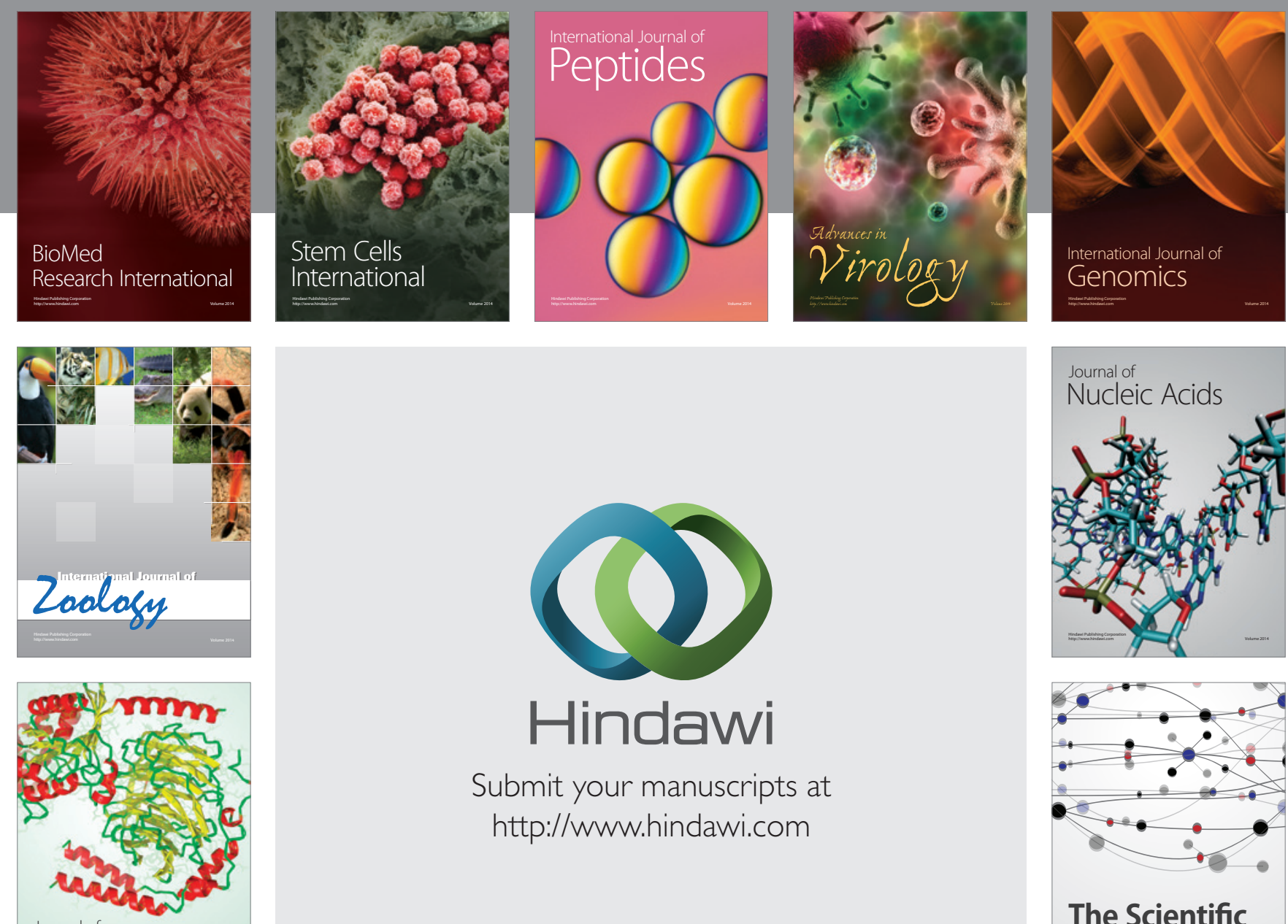

Submit your manuscripts at

http://www.hindawi.com

Journal of
Signal Transduction
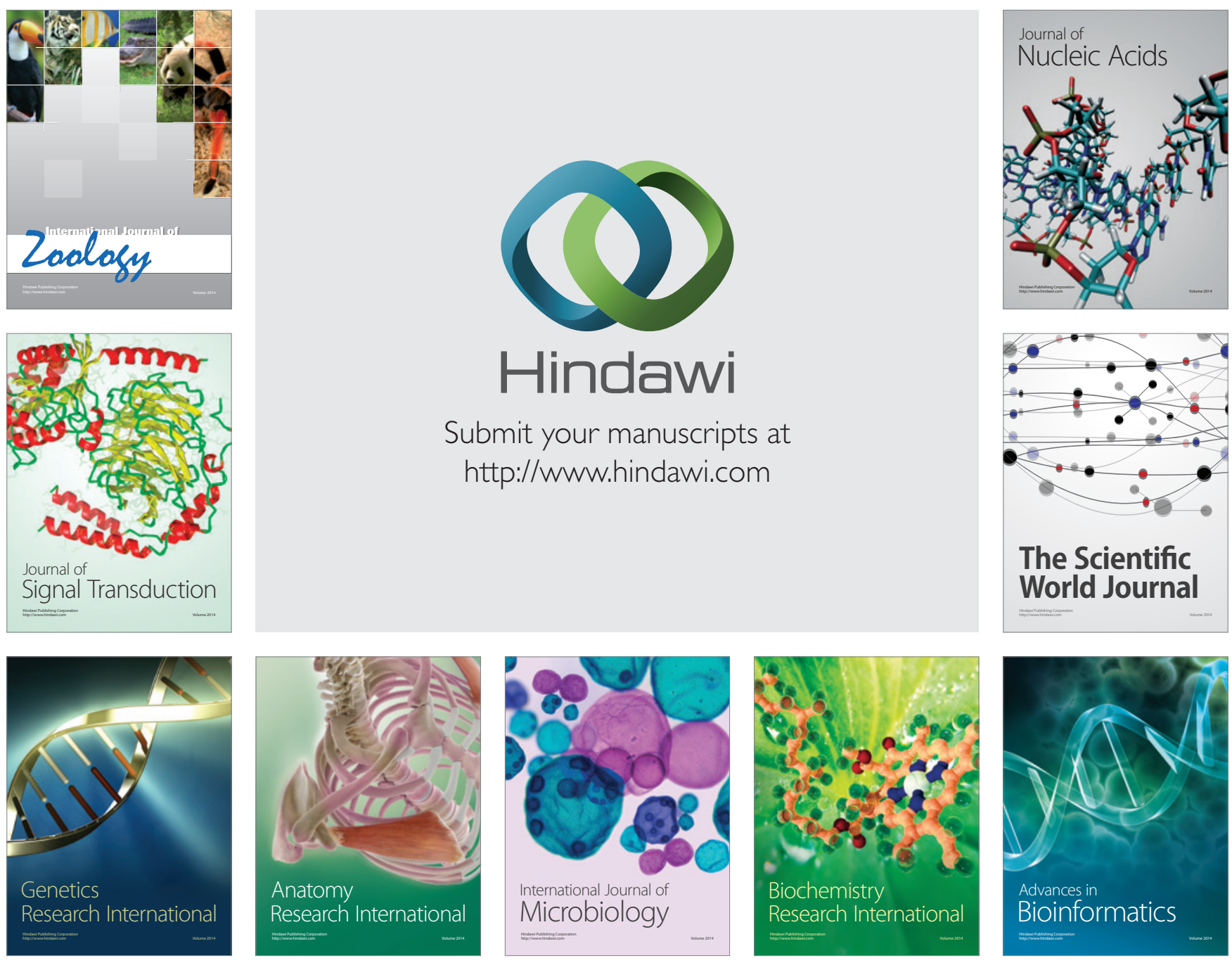

The Scientific World Journal
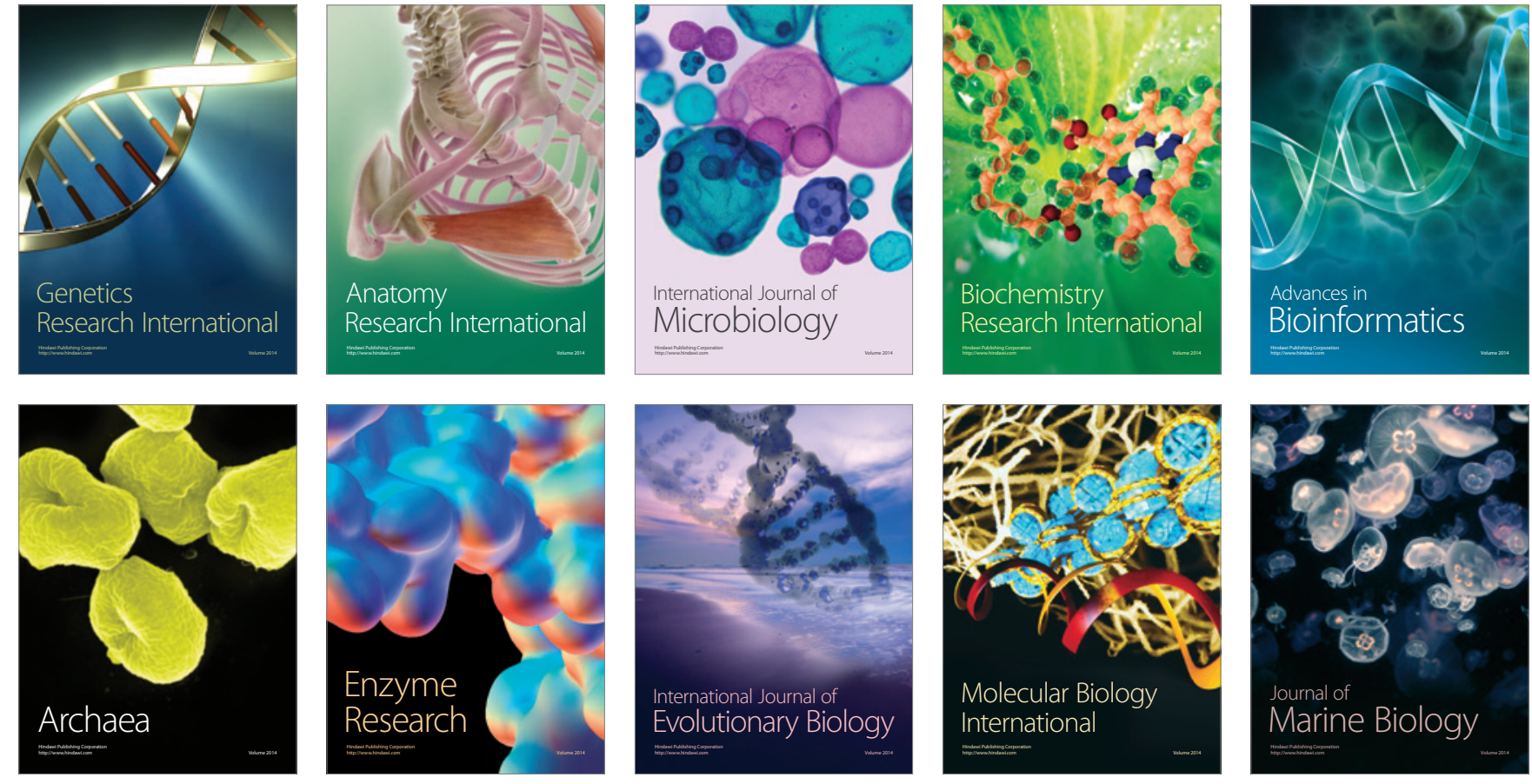